CQUeST-2008-0174

\title{
Anomaly and Hawking radiation from regular black holes
}

\author{
Wontae Kim* \\ Department of Physics and Center for Quantum Spacetime \\ Sogang University, C.P.O. Box 1142, Seoul 100-611, South Korea
}

Hyeonjoon Shin $\dagger^{\dagger}$ and Myungseok Yoon

Center for Quantum Spacetime, Sogang University, Seoul 121-742, South Korea

\begin{abstract}
We consider the Hawking radiation from two regular black holes, the minimal model and the noncommutative black hole. The flux of Hawking radiation is derived by applying the anomaly cancellation method proposed by Robinson and Wilczek. Two regular black holes have the same radiation pattern except for the detailed expression for the Hawking temperature. The resulting flux of the energy-momentum tensor is shown to be precisely the same with the thermal flux from each regular black hole at the Hawking temperature.
\end{abstract}

Keywords : Hawking radiation, anomaly, regular black hole

PACS numbers : 04.62.+v, 04.70.Dy, 11.30.-j

*wtkim@sogang.ac.kr

†hshin@sogang.ac.kr

${ }^{\ddagger}$ younms@sogang. ac . kr 


\section{Introduction}

Hawking radiation is the quantum effect of fields in a classical space-time background with an event horizon [1], and provides an key ingredient to understand the nature of black hole horizon. Since the quantum effect of gravity itself becomes no longer negligible near the black hole horizon, Hawking radiation also provides a basic information in formulating the theory of quantum gravity. Because of its importance, it may be useful to have various interpretations from various different angles, which may lead to some breakthrough in understanding the nature of black hole.

Recently, Robinson and Wilczek [2] came up with a new interpretation about the Hawking radiation. Their proposal is that the Hawking radiation plays the role of preserving general covariance at the quantum level by canceling the diffeomorphism anomaly at the event horizon. It should be noted that the proposal is supposed to be valid in any space-time dimension, contrary to the previous similar work [3] which is restricted to two-dimensional space-time.

For their formulation, Robinson and Wilczek considered the static and spherically symmetric black hole. Elaboration of the original idea and the extensions to more general black holes, the charged and rotating black holes, have been done in [4,5], where it has been shown that Hawking radiation is capable of canceling anomalies of local symmetries at the horizon. In subsequent works [6]- [27], the method of anomaly cancellation has been applied to various black objects in various dimensions even including the black ring. (For a review, see Ref. [28].) As some further elaborations, Hawking fluxes of higher-spin currents have been studied in [29] and some clarifications on the use of anomaly have been given in [30]. All the results until now have given the expected Hawking fluxes and so put the validity of the method on a firmer footing.

In this paper, we consider another interesting class of black hole, the regular black hole, and study the Hawking radiation from it through the anomaly cancellation method. Let us note that one kind of regular black hole has been already explored [8]. It is the wellknown BTZ black hole in three space-time dimensions. What we are concerned about is the four-dimensional regular black hole. In fact, we consider two regular black holes, which are the minimal model [31] and the noncommutative black hole [32]. The noncommutative black hole is especially attractive, since it has a noncommutative parameter and how its effect appears in the Hawking radiation is an interesting issue. Although two regular black holes are surely different to each other, as we will see, these can be dealt with almost simultaneously. 
The organization of this paper is as follows: In the next section, two regular black holes are described. We consider a test real scalar field in the regular black hole background in Sec. 3, and show that, near the horizon, the action for the scalar field reduces to a twodimensional theory in a certain background. In Sec. 4, the flux of the energy-momentum tensor is derived by applying the method of anomaly cancellation to the effective twodimensional theory, and is shown to be the same with the thermal flux at the Hawking temperature. Based on the result, the difference between two regular black holes is discussed.

\section{Regular black holes}

In this section, we introduce two regular black holes, the minimal model and the noncommutative black hole, and explain their basic peculiar properties.

Two regular black holes considered here have the common structure that the geometry is static and spherically symmetric,

$$
d s^{2}=-f(r) d t^{2}+f(r)^{-1} d r^{2}+r^{2} d \Omega_{2}^{2},
$$

where $f(r)$ is a function which vanishes at the event horizon. The explicit form of the function $f(r)$ distinguishes the minimal model and the noncommutative black hole, whose descriptions are given below in order.

\subsection{Minimal model black hole}

Let us suppose a metric which behaves like Schwarzschild black hole at infinity as follows

$$
f(r) \sim 1-\frac{2 m}{r} \text { as } r \rightarrow \infty
$$

where $m$ is the total mass. As for the behavior near the origin or the center, we require the flatness. This may lead to the following choice

$$
f(r) \sim 1-\frac{r^{2}}{\ell^{2}} \quad \text { as } r \rightarrow 0,
$$

where $\ell$ is a positive constant. Actually, this is not an ad hoc choice but is the solution of the Einstein equation with the cosmological constant, $G_{\mu \nu}=-\Lambda g_{\mu \nu}$, near the origin, when the cosmological constant $\Lambda$ is chosen as $\Lambda=3 / \ell^{2}$.

One possible interpolating function, which asymptotes to (2.2) and (2.3) in the corresponding regions, has been derived in [31], and its explicit expression is

$$
f(r)=1-\frac{2 m r^{2}}{r^{3}+2 \ell^{2} m} .
$$




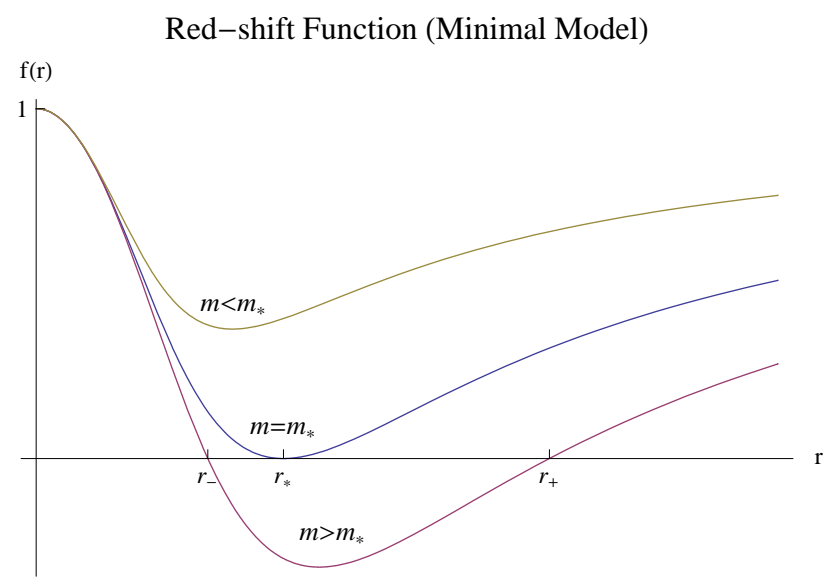

Figure 1: The red-shift function $f(r)$ is regular for all $r$. For $m>m_{*}$ (bottom), there exists two horizons. When $m=m_{*}=3 \sqrt{3} \ell / 4$ (middle), they degenerate. In the case where $m<m_{*}$ (top), there is no horizon.

The geometry (2.1) with the function $f$ of (2.4) has two horizons, the inner horizon $r_{-}$and the outer horizon $r_{+}$, which satisfy the inequality $r_{-} \leq r_{*} \leq r_{+}$. Here $r_{*}$ is a constant defined by $r_{*} \equiv \sqrt{3} \ell$. Because of the presence of the event horizons, we now have a black hole, called the minimal model black hole. The total mass $m$ of the black hole is expressed in terms of the horizons as

$$
m=\frac{r_{ \pm}^{3}}{2\left(r_{ \pm}^{2}-\ell^{2}\right)}
$$

and one can see that it has the minimum value $m_{*}=3 \sqrt{3} \ell / 4$ when $r_{+}=r_{*}$.

We note that the minimal model black hole exists only for $m \geq m_{*}$ as illustrated in Fig. 1. If $m$ is less than $m_{*}$, we do not see any horizon and thus the metric (2.1) does not represent a black hole. One important point is that $r_{*}$ is the minimal value of the event horizon of the minimal model regular black hole. Therefore, one may call it the minimal event horizon. The presence of the minimal horizon is also the case for the noncommutative black hole. In Fig. 2, we illustrate the existence of the minimal mass by plotting the black hole mass with respect to the event horizon. This gives another way of realizing that a black hole does not form if $m<m_{*}$.

\subsection{Noncommutative black hole}

We now turn our attention to the noncommutative black hole. It has been shown that noncommutativity eliminates point-like structures in favor of smeared objects in flat spacetime [33]. The effect of smearing is mathematically implemented by replacing the Dirac-delta function in position space with a Gaussian distribution of the width $\sqrt{\theta}$. In a static and 


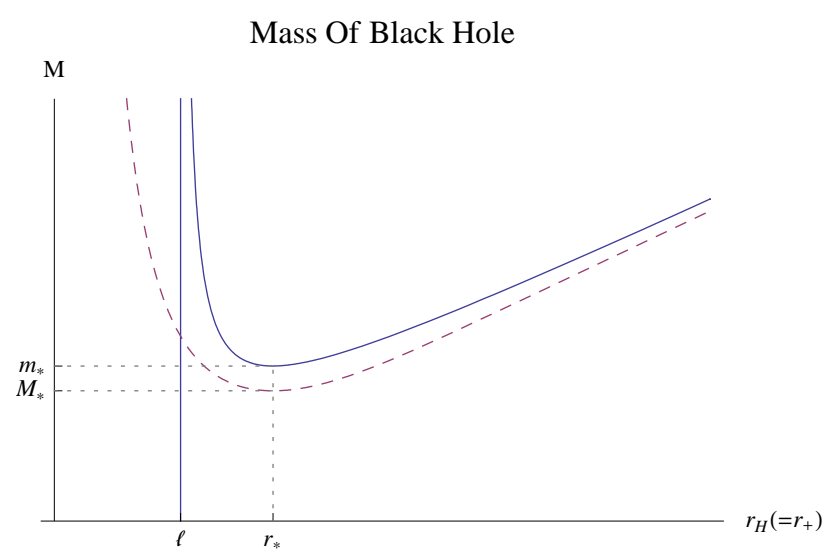

Figure 2: Black hole mass versus the position of the event horizon. The solid and the dashed lines correspond to the minimal model and the noncommutative black holes, respectively. There is minimal mass $m_{*}\left(M_{*}\right)$ to form minimal model black hole (noncommutative black hole). Each black hole has the minimal event horizon for the minimal mass. The minimal event horizons are given by $r_{*}=\sqrt{3} \ell$ for the minimal model black hole and by $r_{*}=2 \alpha \sqrt{\theta}$ for the noncommutative black hole, respectively. In this figure, the minimal horizons are adjusted to have the same value for comparison.

spherically symmetric case, the mass density of a gravitational source is chosen to be [32]

$$
\rho_{\theta}=\frac{M}{(4 \pi \theta)^{3 / 2}} \exp \left(-\frac{r^{2}}{4 \theta}\right)
$$

where $\theta$ is a constant parameter representing noncommutativity and $M$ is the total mass. This mass density implies that the total mass is diffused over the region of linear size $\sqrt{\theta}$.

For a static and spherically symmetric geometry, the energy-momentum tensor is given by $T^{\mu}{ }_{\nu}=\operatorname{diag}\left(-\rho_{\theta}, p_{r}, p_{\perp}, p_{\perp}\right)$. From the conservation law, the radial and the tangential pressure are related to the mass density as $p_{r}=-\rho_{\theta}$ and $p_{\perp}=-\rho_{\theta}-\frac{1}{2} r \partial_{r} \rho_{\theta}$, respectively. Then, from the Einstein equation, we obtain the line element (2.1) with the function

$$
f(r)=1-\frac{4 M}{r \sqrt{\pi}} \gamma\left(\frac{3}{2}, \frac{r^{2}}{4 \theta}\right),
$$

where $\gamma$ is the lower incomplete gamma function,

$$
\gamma(a, z) \equiv \int_{0}^{z} t^{a-1} e^{-t} d t
$$

The function of (2.7) leads to the metric for the noncommutative black hole.

Like the case of the minimal model black hole, we see that there are two horizons, that is, the inner (Cauchy) horizon $r_{C}$ and the outer (event) horizon $r_{H}$, and there exists the minimal mass $M_{*}$ below which no black hole can be formed. These are illustrated in Fig. 3. 


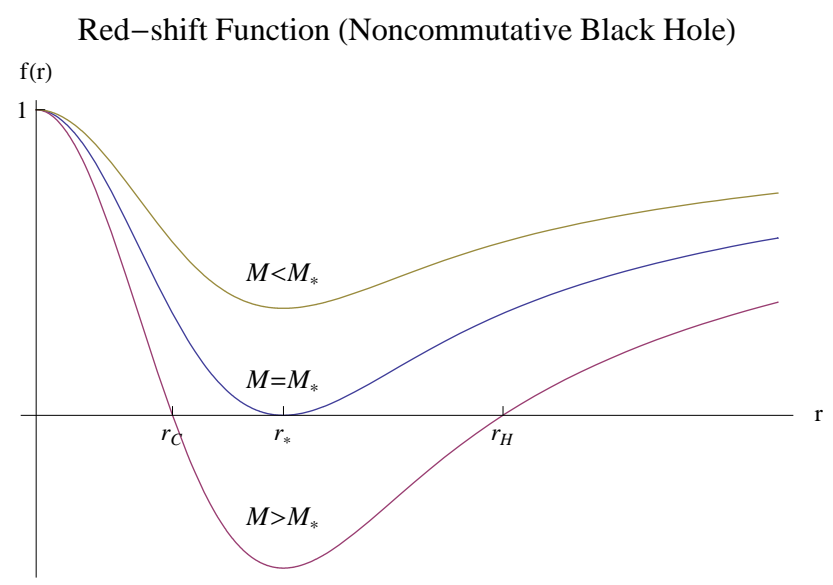

Figure 3: The red-shift function $f(r)$ is regular for all $r$ and its minimum value always appears at $r=r_{*}$. Like the case of the minimal model black hole, there is no horizon for $M<M_{*}$ (top), while two horizons exist for $M>M_{*}$ (bottom) and degenerated one for $M=M_{*} \approx 1.9 \sqrt{\theta}$ (middle), respectively.

Let us now evaluate the minimal mass. First of all, from $f\left(r_{H}\right)=0$, we obtain the relation between the total mass and the event horizon as follows:

$$
M=\frac{r_{H} \sqrt{\pi}}{4 \gamma_{H}},
$$

where $\gamma_{H}=\gamma\left(\frac{3}{2}, \frac{r_{H}^{2}}{4 \theta}\right)$. Based on this relation, we can plot a graph shown in Fig. 2, and see the presence of the minimal mass $M_{*}$. We hope to have the explicit expression of $M_{*}$. However, the analytical evaluation is not an easy task because the relation (2.9) contains an incomplete gamma function. So we take the numerical evaluation of $M_{*}$. Before doing that, it is convenient to hide the noncommutativity parameter $\theta$. This is accomplished by redefining quantities in the red-shift function $f(r)$ as $M \rightarrow M^{\prime}=M /(2 \sqrt{\theta})$ and $r \rightarrow r^{\prime}=$ $r /(2 \sqrt{\theta})$ such that $f(r)$ becomes $1-4 M^{\prime} \gamma\left(3 / 2, r^{\prime 2}\right) /\left(r^{\prime} \sqrt{\pi}\right)$. Then, the redefined red-shift function leads us to have $M^{\prime}=r_{H}^{\prime} \sqrt{\pi} /\left[4 \gamma\left(3 / 2, r_{H}^{\prime 2}\right)\right]$. By solving this and returning back to the original quantities, we get $r_{*}=2 \alpha \sqrt{\theta}$ and $M_{*}=\sqrt{\pi \theta} /\left(4 \alpha^{2} e^{-\alpha^{2}}\right)$, where $\alpha \equiv r_{*}^{\prime}$ is purely a numerical constant determined by

$$
2 \alpha^{3} e^{-\alpha^{2}}=\gamma\left(\frac{3}{2}, \alpha^{2}\right) .
$$

A numerical evaluation gives $\alpha \approx 1.51122$, and thus we finally get $r_{*} \approx 3.02244 \sqrt{\theta}$ and $M_{*} \approx 1.90412 \sqrt{\theta}$. 


\section{Quantum field near the horizon}

We now consider a real free scalar field in the regular black hole background, Eq. (2.1), and investigate its action near the horizon. Although we are dealing with two regular black holes characterized by functions (2.4) and (2.7), the difference between them is not important in this section. It is sufficient to know that both of the regular black holes have the same metric form.

The action for the real scalar field $\varphi$ in the background, Eq. (2.1), is evaluated as

$$
\begin{aligned}
S[\varphi] & =-\int d^{4} x \sqrt{-g} g^{\mu \nu} \partial_{\mu} \varphi \partial_{\nu} \varphi \\
& =\int d t d r r^{2} \int d \vartheta d \phi \sin \vartheta \varphi\left[-\frac{1}{f} \partial_{t}^{2}+\frac{1}{r^{2}} \partial_{r}\left(r^{2} f \partial_{r}\right)+\frac{1}{r^{2}} \nabla_{\Omega}^{2}\right] \varphi
\end{aligned}
$$

where $\nabla_{\Omega}^{2}$ denotes the Laplacian on unit two sphere. If we perform a wave decomposition of $\varphi$ in terms of spherical harmonics $\varphi=\sum_{\ell} \varphi_{\ell} Y_{\ell}(\vartheta, \phi)$, where $\ell$ is the collection of angular quantum numbers of the spherical harmonics and $\varphi_{\ell}$ depends on the coordinates, $t$ and $r$, then we see that the action is reduced to a two-dimensional effective theory with an infinite collection of fields labeled by $\ell$. Next, in order to see what happens near the horizon, it is helpful to take a transformation to the tortoise coordinate $r^{*}$, which, in our case, is defined by $\partial r^{*} / \partial r=1 / f(r)$, and leads to $\int d r=\int d r^{*} f\left(r\left(r^{*}\right)\right)$. If we now go to the region near the horizon, the factor $f\left(r\left(r^{*}\right)\right)$ appears to be a suppression factor vanishing exponentially fast, and thus the terms in the action which do not have some appropriate factor compensating it can be ignored. In the present case, one can easily see that the terms coming from the Laplacian on unit two sphere are suppressed by $f\left(r\left(r^{*}\right)\right)$. We note that the suppression also takes place for the mass term or the interaction terms of $\varphi$ when they are included in the action (3.1).

After all, the action near the horizon becomes

$$
S[\varphi]=\sum_{\ell} \int d t d r r^{2} \varphi_{\ell}\left[-\frac{1}{f} \partial_{t}^{2}+\frac{1}{r^{2}} \partial_{r}\left(r^{2} f \partial_{r}\right)\right] \varphi_{\ell} .
$$

One can check that this action describes an infinite set of massless two-dimensional scalar fields in the following background:

$$
\begin{aligned}
d s^{2} & =-f(r) d t^{2}+\frac{1}{f(r)} d r^{2} \\
\Phi & =r^{2}
\end{aligned}
$$

where $\Phi$ is the two-dimensional dilaton field. 


\section{Anomalies and Hawking fluxes}

In this section, having the two-dimensional effective theory near the horizon (3.2) and the background (3.3), we are going to consider the problem of Hawking radiation following the approach based on the anomaly cancellation proposed in $[2,4]$. Before starting, we would like to note that the two regular black holes distinguished by different $f$ 's, (2.4) and (2.7), can be treated simultaneously. However, the final results will, of course, turn out to be different.

The anomaly approach of [2] begins with an observation that, since the horizon is a null hypersurface, all ingoing (left moving) modes at the horizon can not classically affect physics outside the horizon. This implies that they may be taken to be out of concern at the classical level and thus the effective two-dimensional theory becomes chiral, that is, the theory only of outgoing (right moving) modes. If we now perform the path integration of right moving modes, the resulting quantum effective action becomes anomalous under the general coordinate transformation, due to the absence of the left moving modes. However, such anomalous behaviors are in contradiction to the fact that the underlying theory is not anomalous. The reason for this is simply that we have ignored the quantum effects of the classically irrelevant left moving modes at the horizon. Thus anomalies must be cancelled by including them.

The above argument implies that anomaly is localized at the horizon $r_{H}$. In order to avoid some possible difficulties due to the sharp localization of the anomaly, it is convenient to regard the quantum effective action to be anomalous in an infinitesimal slab, $r_{H} \leq r \leq r_{H}+\epsilon$, which is the region near the horizon. The limit $\epsilon \rightarrow 0$ is taken at the end of the calculation. This leads to a splitting of the region outside the horizon, $r_{H} \leq r \leq \infty$, into two regions,

$r_{H} \leq r \leq r_{H}+\epsilon$ and $r_{H}+\epsilon \leq r \leq \infty$. Then there will be the gravitational anomaly near the horizon, $r_{H} \leq r \leq r_{H}+\epsilon$.

What we are interested in is the problem of determining the flux of the energy-momentum tensor through the cancellation of the gravitational anomaly. Since the region outside the horizon has been divided into two regions, we first write the energy-momentum tensor as a sum

$$
T_{\nu}^{\mu}=T_{\nu(o)}^{\mu} \Theta_{+}(r)+T_{\nu(H)}^{\mu} H(r)
$$

where $\Theta_{+}(r)=\Theta\left(r-r_{H}-\epsilon\right)$ and $H(r)=1-\Theta_{+}(r)$. Among the components of the energymomentum tensor, only the flux in the radial direction, $T_{t}^{r}$, is of concern to us. Apart from 
the near horizon region, $r_{H}+\epsilon \leq r \leq \infty$, it is conserved

$$
\partial_{r} T_{t(o)}^{r}=0
$$

On the other hand, in the near horizon region, $r_{H} \leq r \leq r_{H}+\epsilon$, we have anomalous conservation equation $[2]$ as

$$
\partial_{r} T_{t(H)}^{r}=\partial_{r} N_{t}^{r}
$$

where $N_{t}^{r}=\left(f^{\prime 2}+f f^{\prime \prime}\right) / 192 \pi$. (The prime denotes the derivative with respect to $r$.) The non-vanishing term in the right-hand side is due to the gravitational anomaly for the consistent energy-momentum tensor [34]. Now it is not a difficult task to integrate Eqs. (4.2) and (4.3) and obtain

$$
\begin{aligned}
T_{t(o)}^{r} & =a_{o}, \\
T_{t(H)}^{r} & =a_{H}+\int_{r_{H}}^{r} d r \partial_{r} N_{t}^{r},
\end{aligned}
$$

where $a_{o}$ and $a_{H}$ are integration constants. Here $a_{o}$ is the energy flux which is of our concern.

Next, we consider the variation of quantum effective action $W$ under a general coordinate transformation in the time direction with a transformation parameter $\xi^{t}$ :

$$
\begin{aligned}
-\delta W & =\int d^{2} x \sqrt{-g} \xi^{t} \nabla_{\mu} T_{t}^{\mu} \\
& =\int d^{2} x \xi^{t}\left[\partial_{r}\left(N_{t}^{r} H\right)+\left(T_{t(o)}^{r}-T_{t(H)}^{r}+N_{t}^{r}\right) \delta\left(r-r_{H}-\epsilon\right)\right],
\end{aligned}
$$

where Eqs. (4.1), (4.2) and (4.3) have been used for obtaining the second line. As mentioned before, the full quantum effective action of the underlying theory must be diffeomorphism invariant. The full effective action includes the quantum effects of the ingoing modes near the horizon, whose variation under the general coordinate transformation gives a term canceling the first term of (4.5). For the general covariance of the full quantum effective action, the coefficient of the delta function in Eq. (4.5) is also required to vanish. This requirement leads us to have the following relation.

$$
a_{o}=a_{H}-N_{t}^{r}\left(r_{H}\right)
$$

where the solution Eq. (4.4) has been used. For determining $a_{o}$, the value of the energymomentum flux at the horizon, $a_{H}$, should be fixed. This is done by imposing a condition that the covariant energy-momentum tensor vanishes at the horizon for regularity at the 


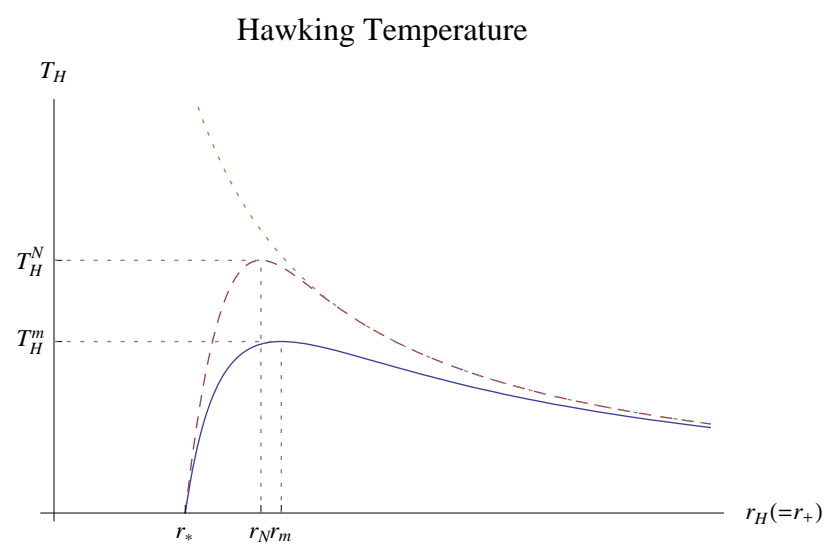

Figure 4: Behavior of Hawking temperature in terms of the position of event horizon. The solid, dashed, and dotted lines correspond to the minimal model, the noncommutative black hole, and the Schwarzschild black hole, respectively. The minimal model (noncommutative) black hole has the maximum Hawking temperature $T_{H}^{m}\left(T_{H}^{N}\right)$ at $r_{+}=r_{m}\left(r_{H}=r_{N}\right)$. One can check that it is the same with that of the Reissner-Nordström black hole with the charge $Q=\sqrt{3} \ell$.

future horizon [5]. Then, from the expression of the covariant energy-momentum tensor $[35,36], \tilde{T}_{t}^{r}=T_{t}^{r}+\frac{1}{192 \pi}\left(f f^{\prime \prime}-2\left(f^{\prime}\right)^{2}\right)$, the condition $\tilde{T}_{t}^{r}\left(r_{H}\right)=0$ gives

$$
a_{H}=\frac{\kappa^{2}}{24 \pi}=2 N_{t}^{r}\left(r_{H}\right)
$$

where $\kappa$ is the surface gravity at the horizon,

$$
\kappa=\left.\frac{1}{2} \partial_{r} f\right|_{r=r_{H}} .
$$

Having the expression of $a_{H}$, the flux of the energy-momentum tensor is finally determined as

$$
a_{o}=N_{t}^{r}\left(r_{+}\right)=\frac{\pi}{12} T_{H}^{2},
$$

where the relation between the Hawking temperature $T_{H}$ and the surface gravity, $T_{H}=\kappa / 2 \pi$, has been used. This is precisely the thermal flux from a regular black hole at the Hawking temperature.

Because the two regular black holes have the same metric form, the expression for the flux of the energy-momentum tensor (4.9) is valid for both of them. However, as mentioned at the beginning in this section, the final results are different for the two black holes. Let us evaluate the Hawking temperature for each black hole based on (4.8). As for the minimal black hole, by using the function $f$ in Eq. (2.4), it is obtained as

$$
T_{H}=\frac{r_{+}^{2}-3 \ell^{2}}{4 \pi r_{+}^{3}} \text {. }
$$


Intriguing thing is that this is the same as that of the Reissner-Nordström black hole when the electric charge of the black hole is given by $Q=\sqrt{3} \ell$. If we turn to another black hole, the noncommutative black hole, the function $f$ in Eq. (2.7) leads us to

$$
T_{H}=\frac{1}{4 \pi r_{H}}\left[1-\frac{M r_{H}^{2}}{\sqrt{\pi} \theta^{3 / 2}} \exp \left(-\frac{r_{H}^{2}}{4 \theta}\right)\right] .
$$

We see that two Hawking temperatures are obviously different and thus lead to the different expressions for the flux. However, we would like to note that they have the similar pattern in terms of the position of the event horizon as shown in Fig. 4. Such pattern may be argued to be a characteristic feature of regular black holes, which is distinguished from, for example, that of the Schwarzschild black hole.

\section{Acknowledgments}

We would like to thank B-H. Lee for useful discussions. This work was supported by the Science Research Center Program of the Korea Science and Engineering Foundation through the Center for Quantum Spacetime (CQUeST) of Sogang University with grant number R11-2005-021.

\section{References}

[1] S. W. Hawking, "Particle Creation By Black Holes," Commun. Math. Phys. 43 (1975) 199 [Erratum-ibid. 46 (1976) 206].

[2] S. P. Robinson and F. Wilczek, "A relationship between Hawking radiation and gravitational anomalies," Phys. Rev. Lett. 95 (2005) 011303 arXiv:gr-qc/0502074.

[3] S. M. Christensen and S. A. Fulling, "Trace Anomalies And The Hawking Effect," Phys. Rev. D 15 (1977) 2088.

[4] S. Iso, H. Umetsu and F. Wilczek, "Hawking radiation from charged black holes via gauge and gravitational anomalies," Phys. Rev. Lett. 96 (2006) 151302 arXiv:hep-th/0602146.

[5] S. Iso, H. Umetsu and F. Wilczek, "Anomalies, Hawking radiations and regularity in rotating black holes," Phys. Rev. D 74 (2006) 044017 arXiv:hep-th/0606018.

[6] K. Murata and J. Soda, "Hawking radiation from rotating black holes and gravitational anomalies," Phys. Rev. D 74 (2006) 044018 arXiv:hep-th/0606069]. 
[7] E. C. Vagenas and S. Das, "Gravitational anomalies, Hawking radiation, and spherically symmetric black holes," JHEP 0610 (2006) 025 [arXiv:hep-th/0606077].

[8] M. R. Setare, "Gauge and gravitational anomalies and Hawking radiation of rotating BTZ black holes," Eur. Phys. J. C 49 (2007) 865 [arXiv:hep-th/0608080]; Q. Q. Jiang, S. Q. Wu and X. Cai, "Hawking radiation from $(2+1)$-dimensional BTZ black holes," Phys. Lett. B 651 (2007) 58 arXiv:hep-th/0701048].

[9] Z. Xu and B. Chen, "Hawking radiation from general Kerr-(anti)de Sitter black holes," Phys. Rev. D 75 (2007) 024041 [arXiv:hep-th/0612261].

[10] S. Iso, T. Morita and H. Umetsu, "Quantum anomalies at horizon and Hawking radiations in Myers-Perry black holes," JHEP 0704 (2007) 068 arXiv:hep-th/0612286.

[11] Q. Q. Jiang and S. Q. Wu, "Hawking radiation from rotating black holes in anti-de Sitter spaces via gauge and gravitational anomalies," Phys. Lett. B 647 (2007) 200 arXiv:hep-th/0701002.

[12] Q. Q. Jiang, S. Q. Wu and X. Cai, "Hawking radiation from the dilatonic black holes via anomalies," Phys. Rev. D 75 (2007) 064029 [Erratum-ibid. 76 (2007) 029904] arXiv:hep-th/0701235.

[13] X. Kui, W. Liu and H. b. Zhang, "Anomalies of the Achucarro-Ortiz black hole," Phys. Lett. B 647 (2007) 482 arXiv:hep-th/0702199.

[14] H. Shin and W. Kim, "Hawking radiation from non-extremal D1-D5 black hole via anomalies," JHEP 0706 (2007) 012 [arXiv:0705.0265 [hep-th]].

[15] J. J. Peng and S. Q. Wu, "Hawking radiation from the Schwarzschild black hole with a global monopole via gravitational anomaly," arXiv:0705.1225 [hep-th]; S. Q. Wu and J. J. Peng, "Hawking radiation from the Reissner-Nordstróm black hole with a global monopole via gravitational and gauge anomalies," Class. Quant. Grav. 24 (2007) 5123 arXiv:0706.0983 [hep-th]]; S. Gangopadhyay, "Hawking radiation in ReissnerNordstróm blackhole with a global monopole via Covariant anomalies and Effective action," arXiv:0803.3492 [hep-th].

[16] Q. Q. Jiang, "Hawking radiation from black holes in de Sitter spaces," Class. Quant. Grav. 24 (2007) 4391 [arXiv:0705.2068 [hep-th]]; Q. Q. Jiang, S. Q. Wu and X. Cai, "Anomalies and de Sitter radiation from the generic black holes in de Sitter spaces," Phys. Lett. B 651 (2007) 65 [arXiv:0705.3871 [hep-th]]. 
[17] B. Chen and W. He, "Hawking Radiation of Black Rings from Anomalies," arXiv:0705.2984 [gr-qc]; U. Miyamoto and K. Murata, "On Hawking radiation from black rings," Phys. Rev. D 77 (2008) 024020 [arXiv:0705.3150 [hep-th]].

[18] W. Kim and H. Shin, "Anomaly Analysis of Hawking Radiation from Acoustic Black Hole," JHEP 0707 (2007) 070 arXiv:0706.3563 [hep-th]].

[19] K. Murata and U. Miyamoto, "Hawking radiation of a vector field and gravitational anomalies," Phys. Rev. D 76 (2007) 084038 [arXiv:0707.0168 [hep-th]].

[20] J. J. Peng and S. Q. Wu, "Can the anomaly cancellation method derive a correct Hawking temperature of a Schwarzschild black hole in the isotropic coordinates ?," arXiv:0709.0044 [hep-th].

[21] J. J. Peng and S. Q. Wu, "Covariant anomaly and Hawking radiation from the modified black hole in the rainbow gravity theory," arXiv:0709.0167 [hep-th].

[22] Z. Z. Ma, "Hawking radiation of black p-branes via gauge and gravitational anomalies," arXiv:0709.3684 [hep-th].

[23] S. Gangopadhyay and S. Kulkarni, "Hawking radiation in GHS and non-extremal D1D5 blackhole via covariant anomalies," Phys. Rev. D 77 (2008) 024038 arXiv:0710.0974 [hep-th]]; S. Gangopadhyay, "Hawking radiation in GHS blackhole, Effective action and Covariant Boundary condition," arXiv:0712.3095 [hep-th].

[24] C. G. Huang, J. R. Sun, X. n. Wu and H. Q. Zhang, "Gravitational Anomaly and Hawking Radiation of Brane World Black Holes," arXiv:0710.4766 [hep-th].

[25] J. J. Peng and S. Q. Wu, "Covariant anomalies and Hawking radiation from charged rotating black strings in anti-de Sitter spacetimes," arXiv:0801.0185 [hep-th].

[26] X. n. Wu, C. G. Huang and J. R. Sun, "On Gravitational anomaly and Hawking radiation near weakly isolated horizon," arXiv:0801.1347 [gr-qc].

[27] S. Q. Wu, J. J. Peng and Z. Y. Zhao, "Anomalies, effective action and Hawking temperatures of a Schwarzschild black hole in the isotropic coordinates," arXiv:0803.1338 [hep-th].

[28] S. Das, S. P. Robinson and E. C. Vagenas, "Gravitational anomalies: a recipe for Hawking radiation," arXiv:0705.2233 [hep-th]. 
[29] S. Iso, T. Morita and H. Umetsu, "Higher-spin currents and thermal flux from Hawking radiation," Phys. Rev. D 75 (2007) 124004 arXiv:hep-th/0701272]; "Fluxes of Higherspin Currents and Hawking Radiations from Charged Black Holes," Phys. Rev. D 76 (2007) 064015 arXiv:0705.3494 [hep-th]]; "Higher-spin Gauge and Trace Anomalies in Two-dimensional Backgrounds," arXiv:0710.0453 [hep-th]; "Hawking Radiation via Higher-spin Gauge Anomalies," Phys. Rev. D 77 (2008) 045007 arXiv:0710.0456 [hepth]].

[30] R. Banerjee and S. Kulkarni, "Hawking Radiation and Covariant Anomalies," Phys. Rev. D 77 (2008) 024018 arXiv:0707.2449 [hep-th]]; "Hawking Radiation, Effective Actions and Covariant Boundary Conditions," Phys. Lett. B 659 (2008) 827 arXiv:0709.3916 [hep-th]]; S. Kulkarni, "Hawking Fluxes, Back reaction and Covariant Anomalies," arXiv:0802.2456 [hep-th].

[31] S. A. Hayward, "Formation and evaporation of nonsingular black holes," Phys. Rev. Lett. 96 (2006) 031103 arXiv:gr-qc/0506126

[32] P. Nicolini, A. Smailagic and E. Spallucci, "Noncommutative geometry inspired Schwarzschild black hole," Phys. Lett. B 632 (2006) 547 arXiv:gr-qc/0510112.

[33] A. Smailagic and E. Spallucci, "Feynman path integral on the noncommutative plane," J. Phys. A 36 (2003) L467 arXiv:hep-th/0307217]; "UV divergence-free QFT on noncommutative plane," J. Phys. A 36 (2003) L517 arXiv:hep-th/0308193.

[34] L. Alvarez-Gaume and E. Witten, "Gravitational Anomalies," Nucl. Phys. B 234 (1984) 269.

[35] W. A. Bardeen and B. Zumino, "Consistent And Covariant Anomalies In Gauge And Gravitational Theories," Nucl. Phys. B 244 (1984) 421.

[36] R. A. Bertlmann and E. Kohlprath, "Two-dimensional gravitational anomalies, Schwinger terms and dispersion relations," Annals Phys. 288 (2001) 137 arXiv:hep-th/0011067. 